\title{
ESTUDIO COMPARATIVO DE TRES MATERIALES DE IMPRESIÓN EN SU CAPACIDAD PARA REPRODUCIR EL MARGEN CERVICAL DE LA PREPARACIÓN MEDIANTE LAS TÉCNICAS DE DOBLE HILO Y COFIA DE TRASFERENCIA.
}

Francisco OJEDA GUTIÉRREZ ${ }^{1}$

Raúl MÁRQUEZ PRECIADO²

Jorge Humberto RAMÍREZ GONZÁLEZ ${ }^{3}$

Nohemí Angélica María ROJAS MUÑOZ ${ }^{4}$

Rita Elizabeth MARTÍNEZ MARTÍNEZ ${ }^{5}$

Paula Rocío SÁNCHEZ ROBLES ${ }^{6}$

Rogelio GONZÁLEZ CORREA ${ }^{7}$

fojeda@uaslp.mx

\section{RESUMEN}

En prótesis fija la integridad marginal o perfección del margen es de suma importancia así como una impresión que copie perfectamente el terminado de la preparación ya que de esto depende la adaptabilidad de la prótesis que permita la supervivencia de la restauración y el mantenimiento de la salud periodontal en la boca del paciente. En este estudio se compara la capacidad de tres materiales de impresión ( 2 siliconas por adición y 1 poliéter) para copiar el margen cervical de la preparación mediante las técnicas de doble hilo y cofia de trasferencia. Se utilizaron 96 primeros molares inferiores izquierdos divididos en 6 grupos de 16 c/u se restauraron los dientes con ionómero de vidrio, resina o poste colado según fuera el caso, se prepararon los dientes con terminación en chamfer, el grupo 1: técnica de doble hilo con silicona por adición (Silicona 1), grupo 2: técnica de doble hilo con poliéter, grupo 3: técnica de cofia de transferencia con silicona (Silicona 1), grupo 4: técnica de cofia de transferencia con poliéter, grupo 5: Técnica de doble hilo con silicona (Silicona 2), grupo 6: Técnica de cofia con silicona (Silicona 2). Posteriormente fueron observados en el estereomicroscopio para evaluar la cantidad de burbujas e irregularidades en el margen

1 Dr. Francisco Ojeda Gutiérrez, Departamento de Estomatología Integral, Facultad de Estomatología, Universidad Autónoma de San Luis Potosí, San Luis Potosí, México.

2 Dr. Raúl Márquez Preciado, Departamento de Odontopediatría, Facultad de Estomatología, Universidad Autónoma de San Luis Potosí, San Luis Potosí, México.

3 Dr. Jorge Humberto Ramírez González, Laboratorio de Ciencias Odontológicas, Maestría en Ciencias Odontológicas, Universidad Autónoma de San Luis Potosí. San Luis Potosí, México

4 Dra. Nohemí Angélica María Rojas Muñoz, Departamento de Estomatología Integral, Facultad de Estomatología, Universidad Autónoma de San Luis Potosí, San Luis Potosí, México.

5 Dra. Rita Elizabeth Martínez Martínez, Maestría en Ciencias Odontológicas, Facultad de Estomatología, Universidad Autónoma de San Luis Potosí, San Luis Potosí, México.

6 Dra. Paula Rocío Sánchez Robles, Departamento de Estomatología Integral, Facultad de Estomatología, Universidad Autónoma de San Luis Potosí, San Luis Potosí, México.

7 Dr. Rogelio González Correa, Departamento de Estomatología Integral, Facultad de Estomatología, Universidad Autónoma de San Luis Potosí, San Luis Potosí, México. 
OJEDA GUTIÉRREZ F, MARQUEZ PRECIADO R, RAMIREZ GONZÁLEZ JH, ROJAS MUÑOZ NAM, MARTÍNEZ MARTÍNEZ RE, SÁNCHEZ ROBLES PR, GONZALEZ CORREA R. Estudio comparativo de tres materiales de impresión en su capacidad para reproducir el margen cervical de la preparación mediante las técnicas de doble hilo y cofia de trasferencia.. Rev. Odontol. Univ. Cid. São Paulo 2018 abr/jun 30(2) 157-168

ISSN 1983-5183

cervical según la escala de Jokstad. Donde la prueba estadística de Chi-cuadrada con un nivel de confianza de $<0.05$ no mostró diferencia estadística significativa entre las técnicas de impresión de doble hilo y la de cofia de trasferencia, independientemente del material usado. Aunque numéricamente la técnica de doble hilo se comportó mejor que la técnica de cofia por lo que se sugiere su uso.

PALABRAS CLAVE: MATERIALES DE IMPRESIÓN DENTAL - SILICONAS, TÉCNICA DE IMPRESIÓN DENTAL.

\title{
COMPARATIVE STUDY OF THREE IMPRESSION MATERIALS IN THEIR CAPACITY TO REPRODUCE THE CERVICAL MARGIN OF PREPARATION USING TECHNIQUES OF COPING AND DOUBLE CORD
}

\begin{abstract}
In fixed prosthesis, the integrity of perfection of the margin is of utmost importance, as well as an impression that perfectly copies the finish of the preparations, since the adaptability of the prosthesis that ensures survival of the restoration and periodontal health depends on this. This study compares the capacity of 3 different materials ( 2 silicones addition and 1 polyether) to copy the cervical margin of the preparation in the impression between the techniques of double cord and coping. We used 96 lower left molars divided into 6 groups of 16 each. The teeth were restored with glass ionomer, resin or cast post and core depending on the case, the margins were finished in a chamfer (group 1), double thread with addition silicone (group 2), double thread with polyether, (group 3), coping with PVS, (group 4), coping with polyether, (group5) double cord with PVS (group 6), coping With PVS. They were subsequently evaluated in a stereomicroscope to observe the number of bubbles and irregularities in the cervical margin according to the Jockstad scale, where the Chi-square test with a confidence level of $<0.05$ showed no statistically significant difference between impression techniques and coping regardless of material used. Although numerically the double-thread technique behaved better than coping technique, so it is suggested its use.
\end{abstract}

KEY WORDS: DENTAL IMPRESSION MATERIALS - SILICONES - DENTAL IMPRESSION TECHNIQUE.

\section{INTRODUCCIÓN}

La preparación protésica puede ser definida como un proceso de desgaste selectivo de esmalte y/o dentina en cantidades y áreas predeterminadas, con la finalidad de crear espacio para la prótesis fija. Sus objetivos están orientados en primer lugar a devolver la función y estética y en segundo lugar a mantener las condiciones de salud logradas con los procedimientos previos.

En prótesis fija la integridad marginal o perfección del margen es de suma importancia ya que los márgenes adaptados a la línea terminal permiten la supervivencia de la prótesis en el medio bucal, el tipo de línea cervical influye directamente en la calidad de la restauración y su localización establece la salud periodontal' , ya que, el éxito de cualquier restauración vaciada depende de la adaptación de ella al diente, un margen deficiente conduce a la retención de placa resultando en gingivitis y filtración marginal siendo un factor que conduce a caries secundaria, sensibilidad, recesión gingival, disolución del cemento y la pérdida de la restauración. ${ }^{1}$ Por esto la separación gingival es sumamente importante ${ }^{2}$ ya que provoca: retracción del tejido gingival y expone temporalmente los márgenes ${ }^{3}$ de la preparación tallada permitiendo una buena impresión en negativo de la terminación cervical ${ }^{4}$, para que esto se logre se tiene que utilizar una técnica de separación de la encía adecuada ${ }^{5}$, permitiendo obtener una impresión precisa de los detalles 
OJEDA GUTIÉRREZ F, MARQUEZ PRECIADO R, RAMIREZ GONZÁLEZ JH, ROJAS MUÑOZ NAM, MARTÍNEZ MARTÍNEZ RE, SÁNCHEZ ROBLES PR, GONZALEZ CORREA R. Estudio comparativo de tres materiales de impresión en su capacidad para reproducir el margen cervical de la preparación mediante las técnicas de doble hilo y cofia de trasferencia.. Rev. Odontol. Univ. Cid. São Paulo 2018 abr/jun 30(2) 157-168

ISSN 1983-5183

marginales y un resultado estético óptimó6.

Las técnicas de retracción gingival más comúnmente usadas son: La de cofia ${ }^{7}$ son provisionales realizados con acrílico, monómero-polímero ${ }^{4}$. El rebasado se realizó con acrílico duralay el cual protege la cofia del desplazamiento y distorsión, y causa menos daño al periodonto tanto en el diseño como en la colocación de la restauración ${ }^{8}$, facilitando la visualización de los márgenes cervicales y el surco gingival ya que entra en la unión de encía y diente, esta funciona como una guía o llave para el correcto reposicionamiento de la cofia y prevee el desplazamiento en la toma de impresión con la cucharilla, permitiendo únicamente tomar la impresión del órgano dentario a tratar ${ }^{9}$.

Técnica de doble hilo:Como su nombre lo indica, se usan dos hilos, primero el delgado y posteriormente el grueso, esta técnica es dada por el empaquetamiento del hilo con el instrumento empacador dirigido paralelo a la superficie de la raíz hasta sentir resistencia permitiendo la separación temporal del tejido gingival $^{3,10}$, exponiendo la superficie del tallado, se deja reposar 5 min aproximadamente, se retira el segundo hilo y posteriormente se toma la impresión ${ }^{4}$, cuando el hilo va impregnado de una solución astringente que causa isquemia transitoria haciendo que el tejido gingival se contraiga y ayuda a controlar el exudado del fluido gingival ${ }^{4,5,11}$. El uso inapropiado de la solución puede dar como resultado una recesión gingival, impresiones pobres, frustración y aumento en el costo por repeticiones ${ }^{10}$.

Curetaje rotatorio: El Curetaje gingival rotatorio es un procedimiento quirúrgico que intenta eliminar el tejido enfermo de la pared interna del surco hasta dejarlo sano ${ }^{12}$.

Electrocirugía: Se usa para ensanchar el surco gingival y controlar la hemorragia deberá controlar primero con presión y/o productos químicos para poder a continuación sellar los vasos ${ }^{13}$.

\section{MATERIALES Y MÉTODOS}

Previamente se calibró a la alumna en cuanto a: las preparaciones en un modelo de resina con dientes naturales en la utilización y manipulación de materiales y técnicas de impresión (colocación de hilo, realización de cofia y rebasado de la misma ${ }^{14}$, posteriormente se seleccionaron 96 primeros molares inferiores izquierdos con 1 paciente por cada molar por lo que fueron 96 impresiones irrepetibles, se le realizó una historia clínica al paciente y se le hizo firmar una carta de consentimiento informado donde aceptó participar en el estudio, a todos se les tomó modelos de estudio y radiografía periapical del O.D. a tratar, se dividieron en 6 grupos de 16 O.D. c/u, se le realizó una impresión con spidex pesado (Speedex Putty, Coltène Whaledent, Ohio/USA) en el O.D. 36 para utilizarla como guía en el provisional, se reconstruyó con resina (3M ESPE, Filtek Z 250 XT Nano Hybrid Universal Restorative, Conway Avenue St. Paul. MN 55144-1000 USA), ionómero de vidrio (Vitremer 3M ESPE Dental Products 2510 Conway, Avenue St. Paul, MN 55144-1000 USA) o endoposte colado cementado (Aleación de metal Aluminio-Cobre-Paladio Regiocast ALPRODEN, Aleaciones y Productos Dentales S.A. de C.V., Monterey, NL, México). Según era el caso (Figura 1 A-D). Los 96 O.D. se prepararon con terminación en forma de chamfer ${ }^{4}$, con una fresa troncocónica de punta redondeada (F4R, Le Blond, A\&M Instrument. inc) ${ }^{14}$ comenzando por la cara distal, lingual, mesial y vestibular, posteriormente se preparó la cara oclusal formando un surco principal y dos vertientes (vestibular y lingual). 

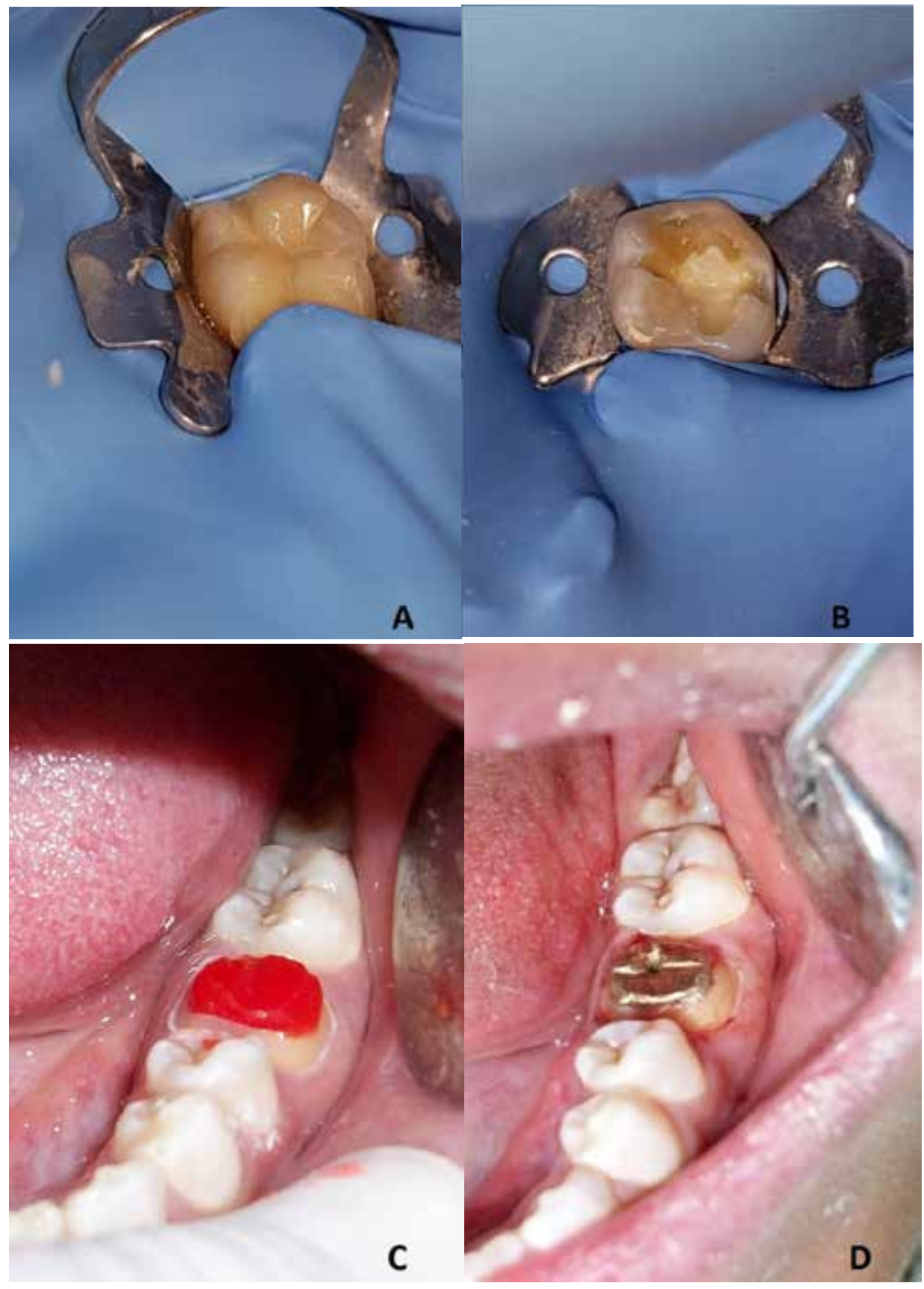

Figura 1. Reconstrucción del órgano dentario previo a la impresión A), Resina, B), lonómero de vidrio, C), Impresión del poste con duralay D), Colocación y cementado del poste.

Grupo I- (Silicona 1) Se realizó la impresión con técnica de doble hilo (Figura 2 A, B), la cual consistió en aislar la zona relativamente con torundas de algodón para mantenerla seca, se cortaron unos tramos de hilo (Ultrapak, Ultradent Products, inc. South Jordan USA) 00 y 0 de aprox. 5.0mm cada uno, se colocó primero el hilo de menor diámetro en forma de "u", con un instrumento de punta roma (Guyer, Hu-Fredy) sin utilizar ningún tipo de sustancia hemostática, se fue empacando en forma apical entre la encía y el diente con una presión ligera comenzando por la cara mesial, lingual, distal, terminando en vestibular ${ }^{4}$. Se tomó la impresión con silicona por adición, primero con material pesado (Express TM Penta, Germany by 3M 
ESPE AG, dental products) dispensado de la maquina penta (3M ESPE PentamixTM 2) a la cucharilla completa de plástico con movimientos de vaivén simulando una "oruga", posteriormente se colocó el segundo hilo como antes se mencionó, se eliminaron los espacios interproximales de la impresión con un bisturí, se retiró el segundo hilo dejando el primero, se secó el surco crevicular con aire de la jeringa triple se prosiguió con material ligero (ExpressTM XT Light Body, Germany by 3M ESPE AG, dental products) con la pistola dispensadora en el surco seco, posteriormente se llevó el resto del material ligero en la cucharilla y se sitúo en la boca del paciente y posteriormente retirar la impresión?. (Figura $\mathbf{2 ~ C ) ~}$

Grupo II - Se realizó la toma de impresión con técnica de doble hilo y poliéter pesado (ImpregumTM Penta, Germany by 3M ESPE AG, dental products), posteriormente poliéter ligero (ImpregumTM Soft, Germany by $3 \mathrm{M}$ ESPE AG, dental products) de la misma forma que se mencionó anteriormente. (Figura 2 D)
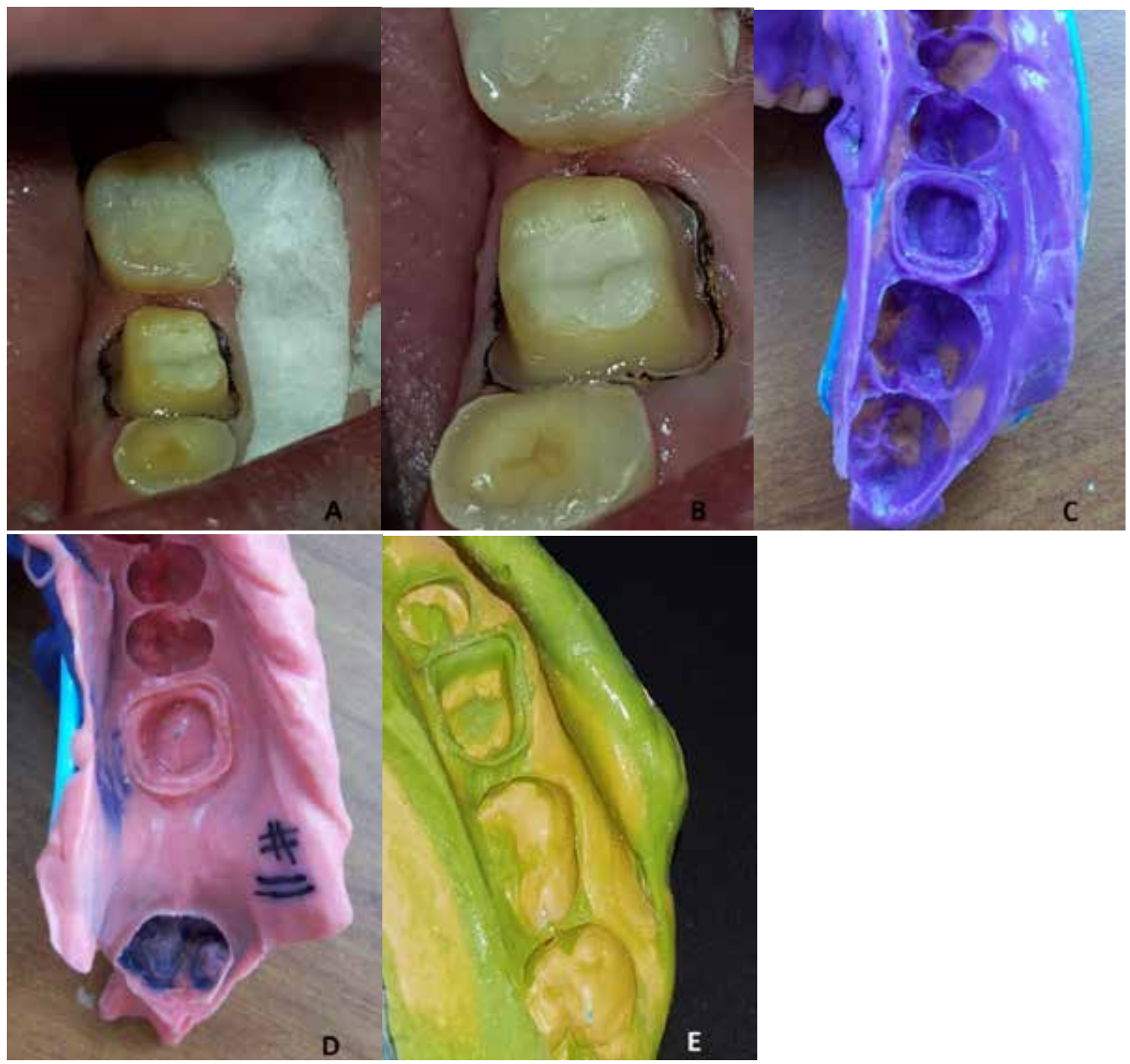

Figura 2. Técnica de doble hilo. A), Colocación del hilo delgado, B), Colocación del segundo hilo grueso intra crevicular con un instrumento de punta roma. C), Toma de Impresión con Express Penta, D), Toma de Impresión con Poliéter, E), Impresión con silicona por adición HydroXtreme. 
OJEDA GUTIÉRREZ F, MARQUEZ PRECIADO R, RAMIREZ GONZÁLEZ JH, ROJAS MUÑOZ NAM, MARTÍNEZ MARTÍNEZ RE, SÁNCHEZ ROBLES PR, GONZALEZ CORREA R. Estudio comparativo de tres materiales de impresión en su capacidad para reproducir el margen cervical de la preparación mediante las técnicas de doble hilo y cofia de trasferencia.. Rev. Odontol. Univ. Cid. São Paulo 2018 abr/jun 30(2) 157-168

ISSN 1983-5183

Grupo III - (Silicona 1). La retracción gingival se realizó con cofia de trasferencia (Figura 3 A-C), la cual consiste en tomar una impresión con alginato (Kromopan lescod made in Italy) del O.D. a tratar ya preparado, se vació en yeso blanco (Yeso Piedra, Whip Mix, Kentucky USA.) posteriormente se realizó un surco de aproximadamente $2 \mathrm{~mm}$ por debajo y alrededor del diente preparado, se impregnó separante para yeso como aislante, se mezcló acrílico y monómero-polímero autocurable (Nic Tone, MDC dental, Zapopan Jal México ) en un vaso de dappen, una pequeña cantidad de la mezcla se colocó en el O.D preparado, ya que polimerizó se retiró del modelo, con un lápiz se marcó el contorno de la cofia, se recortó y se abocardó con fresón de carburo y fresa de bola para baja velocidad, haciendo retención con relieves en la cara superior se revisó en el diente para asegurar que separara la encía y entrara sin dificultad, para el rebasado se utilizó acrílico duralay polvo y líquido (Duralay, Relianc, Worth Illinois USA) se colocó en el borde de la preparación con un pincel o una espátula, se insertó la cofia, con ayuda de una espátula o cola de castor se empujó el duralay para que lograra impresionar correctamente la terminación cervical y separara la encía, se evitaran movimientos bruscos ya que podría traumatizar el tejido, conforme pasó el tiempo de polimerización se desinsertó la cofia y se volvió a colocar para evitar que se pegara al diente, después de polimerizado se retiró de la cavidad bucal, se recortó nuevamente y se abocardó, si era necesario se realizaba un segundo o tercer rebasado hasta que entrara completamente en el surco y pudiera separar el tejido ${ }^{15}$, se tomó la impresión con polivinil pesado (Express TM Penta, Germany by 3M ESPE AG, dental products) dispensado de la maquina penta (3M ESPE PentamixTM 2 en toda la arcada con la cofia colocada en el O.D, se retiró de la cavidad bucal, se desinsertó la cofia de la cucharilla, se eliminaron los espacios interproximales de la cucharilla con bisturí para realizar espacio para el material ligero, se colocó polivinil ligero(ExpressTM XT Light Body, Germany by 3M ESPE AG, dental products) dentro de ella con una espátula y en el resto de la cucharilla se volvió a llevar a la cavidad, nos esperamos aprox 5 min para retirar la cucharilla con la impresión. (Figura 3 D)

Grupo IV - La retracción gingival se realizó con técnica de cofia de trasferencia y la toma de impresión fue con poliéter pesado. ImpregumTM Penta, Germany by 3M ESPE AG, dental products), y posteriormente poliéter ligero (ImpregumTM Soft, Germany by 3M ESPE AG, dental products). (Figura 3 E)

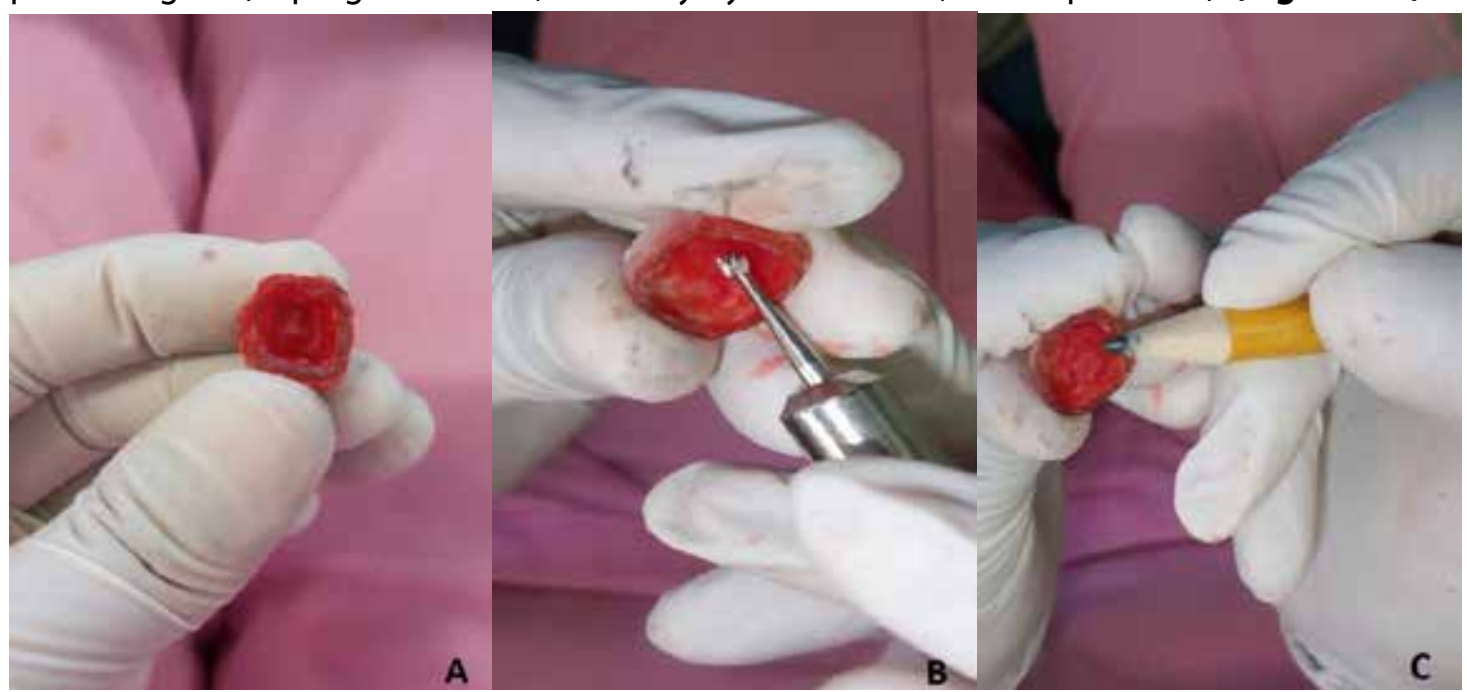




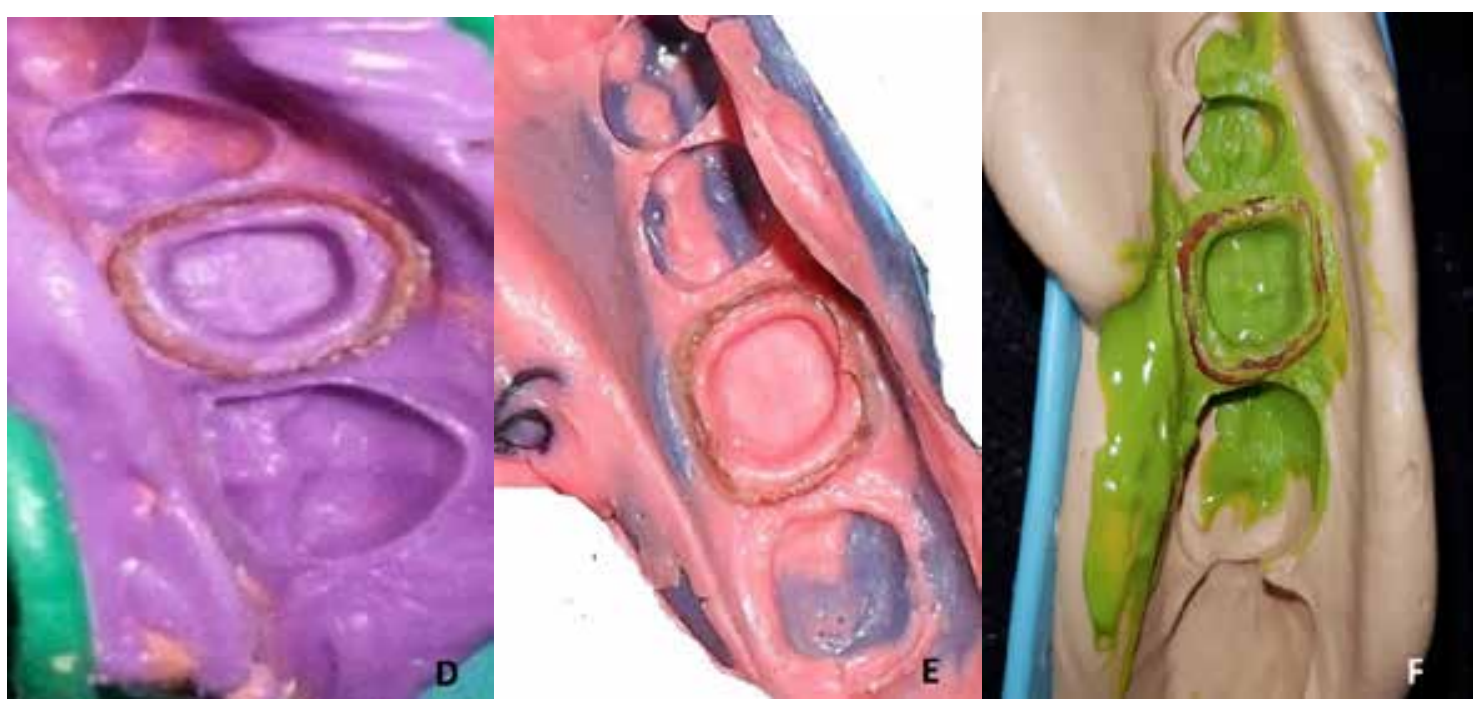

Figura 3. Técnica de cofia de transferencia. A), Retiro de la cofia de la preparación, B), Abocardado de la cofia con fresón de bola de carburo, C), Marcado del contorno con lápiz de grafito, D), Toma de impresión con Express Penta, E), Toma de impresión con Poliéter, F), Toma de impresión con silicona HydroXtreme.

Grupo V - (Silicona 2). Se realizó la impresión con técnica de doble hilo, bajo las mismas condiciones del primer grupo se utilizó la primera impresión con siliconas por adición (HydroXtreme COLTÉNE/ WHALEDENT, SWITZERLAND) en masilla llenando la cucharilla y enseguida el material ligero con la pistola dispensadora en el surco y la cucharilla de ahí a la boca del paciente. (Figura 2 E)

Grupo VI - (Silicona 2). Se usó misma técnica de cofia de trasferencia, se tomó la impresión con siliconas por adición (HydroXtreme COLTÉNE/WHALEDENT, SWITZERLAND ) en toda la arcada con la cofia colocada en el O.D, se retiró de la cavidad bucal, se desinsertó la cofia de la cucharilla, se eliminaron los espacios interproximales de la cucharilla con bisturí para realizar espacio para el material ligero, que se colocó con la pistola dispensadora (HydroXtreme COLTÉNE/WHALEDENT, SWITZERLAND) dentro de la cucharilla y la cofia y obtener la impresión. (Figura 3 F)

Posteriormente todas las muestras fueron examinadas en la nitidez de la preparación gingival en toda su circunferencia al estereomiscroscopio (SZ-PT Olympus, Japón) con un zoom de 10X, por un evaluador externo al estudio el cual clasificó las impresiones y les dió un valor a cada una tomando de referencia la siguiente escala según Jokstad ${ }^{16}$ :

\begin{tabular}{|l|l|}
\hline Perfecto-0: & Se refiere a que no hay ninguna burbuja de aire en la línea de terminado cervical. \\
\hline Buena-1: & Se refiere a una burbuja de aire en una sola área, línea de terminado cervical continua. \\
\hline No está mal-2: & $\begin{array}{l}\text { Se refiere a una sola burbuja de aire en una sola área, línea de terminado cervical interrum- } \\
\text { pido. (Figura 4 A-B). }\end{array}$ \\
\hline Mal-3: & Se refiere a que hay más de una burbuja de aire en la línea de terminación cervical. \\
\hline
\end{tabular}




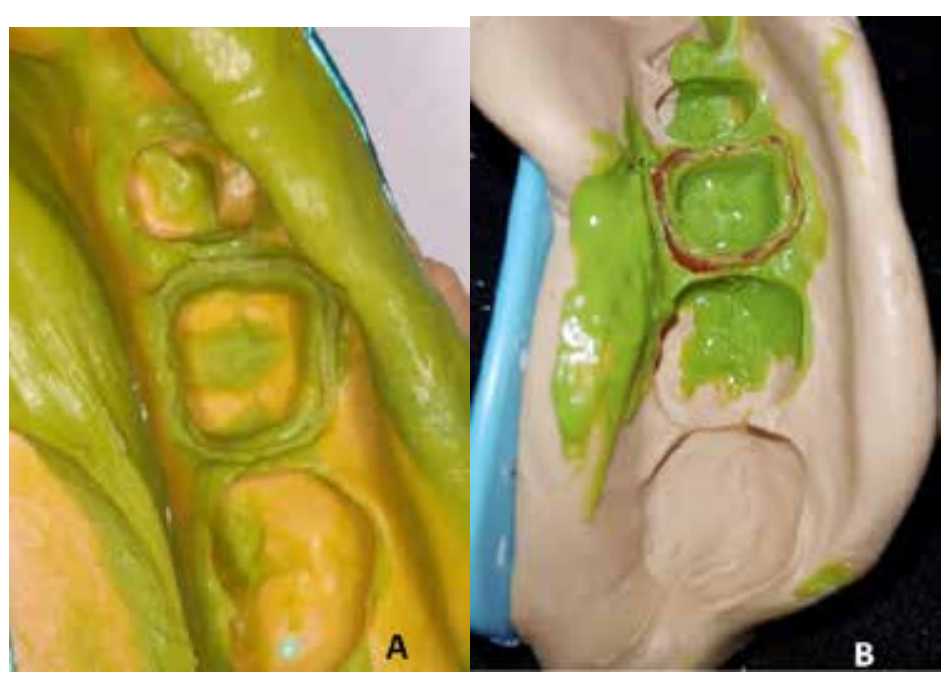

Figura 4. Según la escala de Jockstad se refiere a la interpretación de "no está mal "2. Representa una burbuja de aire en una sola línea, con terminado cervical interrumpido. A) Burbuja en la cara lingual de la preparación. B) Interrupción en el terminado cervical de la cara mesial.

\section{ANÁLISIS ESTADÍSTICO}

El análisis estadístico fue llevado a cabo con el software GraphPad Prism versión 7.0 (GraphPad Software, Inc. La Jolla, CA, USA). La prueba de chi-cuadrada ( $\left.\mathrm{X}^{2}\right)$ con un nivel de confianza de $\mathrm{P}<0.05$ fue utilizada para comparar las frecuencias de la escala de Jokstad entre todos los grupos de estudio

\section{RESULTADOS}

Se incluyeron 6 grupos de 16 muestras cada uno por lo que en su totalidad fueron 96 muestras evaluadas. El Cuadro 1 y la Figura 5 muestran la comparación de la frecuencia y porcentaje de los valores de la escala de Jokstad entre todos los grupos de estudio, se destaca que todos los grupos de estudio presentaron la misma frecuencia (31.3\%) en la escala 0 (Perfecto). Se puede observar que todos los grupos presentaron una mayor frecuencia en la escala 1 (Buena) resaltando que los grupos que numéricamente mejor se comportaron fueron el 1 y el 5 en contraste con el grupo 3, además se observó que en la escala 2 (No está mal) los grupos 3 y 6 sobresalieron numéricamente en comparación del resto de los mismos, por otro lado, se encontró una menor frecuencia en la escala 3 (Mal) resaltando que los grupos de estudio 1,5 y 6 se comportaron mejor numéricamente que los grupos 2,3 y 4, sin embargo no se encontraron diferencias estadísticamente significativas en ninguno de las escalas evaluadas. 
OJEDA GUTIÉRREZ F, MARQUEZ PRECIADO R, RAMIREZ GONZÁLEZ JH, ROJAS MUÑOZ NAM, MARTÍNEZ MARTÍNEZ RE, SÁNCHEZ ROBLES PR, GONZALEZ CORREA R. Estudio comparativo de tres materiales de impresión en su capacidad para reproducir el margen cervical de la preparación mediante las técnicas de doble hilo y cofia de trasferencia.. Rev. Odontol. Univ. Cid. São Paulo 2018 abr/jun 30(2) 157-168

ISSN 1983-5183

\begin{tabular}{|c|c|c|c|c|}
\hline \multirow{3}{*}{$\begin{array}{c}\text { Grupo de } \\
\text { estudio }\end{array}$} & \multicolumn{4}{|c|}{ Escala de Jokstad } \\
\cline { 2 - 5 } & $\begin{array}{c}\text { Perfecto } \\
\mathbf{0}\end{array}$ & $\begin{array}{c}\text { Buena } \\
\mathbf{1}\end{array}$ & $\begin{array}{c}\text { No está mal } \\
\mathbf{2}\end{array}$ & $\begin{array}{c}\text { Mal } \\
\mathbf{3}\end{array}$ \\
\cline { 2 - 5 } & & \multicolumn{4}{|c|}{ Frecuencia } & $\mathbf{1} \%)$ \\
\cline { 2 - 5 } & $5(31.3)$ & $9(56.3)$ & $2(12.4)$ & 0 \\
\cline { 2 - 5 } $\mathbf{1}$ & $5(31.3)$ & $8(50.0)$ & $2(12.5)$ & $1(6.2)$ \\
\cline { 2 - 5 } $\mathbf{2}$ & $5(31.3)$ & $7(43.7)$ & $3(18.8)$ & $1(6.2)$ \\
\cline { 2 - 5 } $\mathbf{4}$ & $5(31.3)$ & $8(50.0)$ & $2(12.5)$ & $1(6.2)$ \\
$\mathbf{5}$ & & $9(56.3)$ & $2(12.4)$ & 0 \\
$\mathbf{6}$ & $5(31.3)$ & $8(50)$ & $3(18.2)$ & 0 \\
\cline { 2 - 5 } $\mathbf{P}$ & $5(31.3)$ & 0.494 & 0.469 & 0.313 \\
\cline { 2 - 5 } & - & & & \\
\hline
\end{tabular}

Cuadro 1. Con referencia en la escala de Jokstad se destaca que todos los grupos de estudio presentaron la misma frecuencia en la escala de 0 (Perfecto).

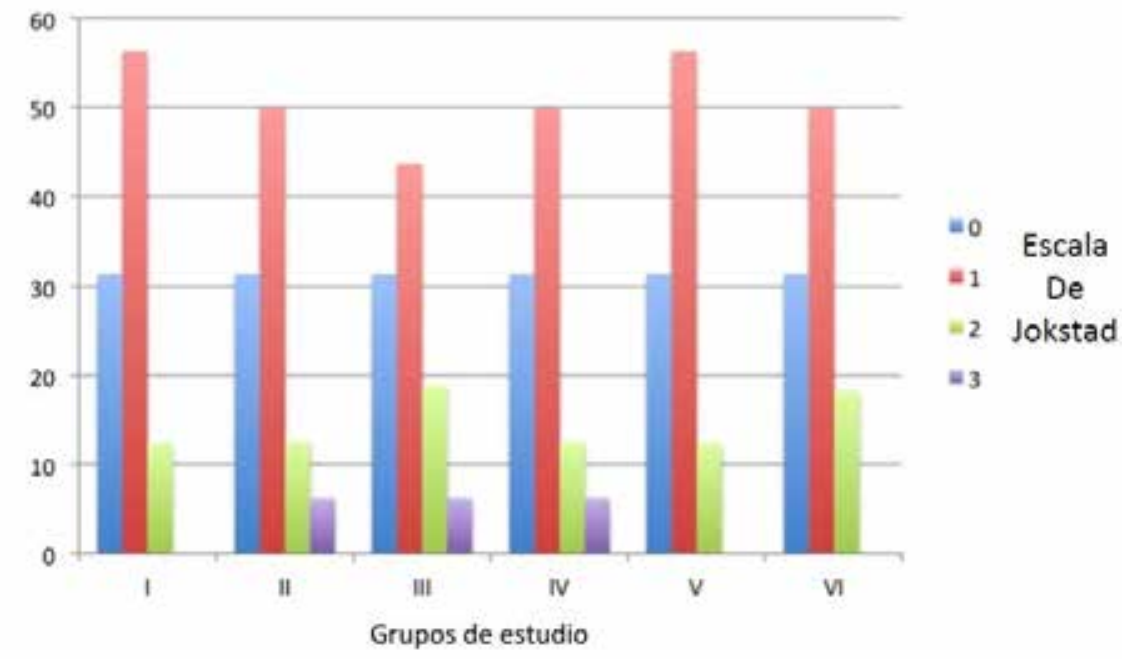

Figura 5. Muestra la comparación de la frecuencia y porcentaje de los valores en la escala de Jokstad.

\section{DISCUSIÓN}

Una impresión puede ser definida como el acto de reproducir en negativo una determinada superficie, un material en estado plástico con propiedades de escurrimiento es colocado sobre una determinada región de la boca con el objetivo de copiar los detalles existentes ${ }^{9}$ por lo que existen diversos tipos de materiales de impresión como son los hidrocoloides, mercaptanos, poliéter y siliconas, los cuales son clasificados de acuerdo a su tipo de polimerización en tipo I por condensación y tipo II por adición $n^{17}$. Aunque estos últimos son los más utilizados para prótesis fija por sus ventajas como son tiempo de trabajo adecuado, manipulación manual, resistencia al desgarrado, estabilidad dimensional, excelente fluidez y buena recuperación elástica' ${ }^{18}$. La técnica más utilizada actualmente es la de doble hilo, dentro de las ventajas es que tiene mayor efectividad en la separación entre la encía y el diente para una vista más nítida 
OJEDA GUTIÉRREZ F, MARQUEZ PRECIADO R, RAMIREZ GONZÁLEZ JH, ROJAS MUÑOZ NAM, MARTÍNEZ MARTÍNEZ RE, SÁNCHEZ ROBLES PR, GONZALEZ CORREA R. Estudio comparativo de tres materiales de impresión en su capacidad para reproducir el margen cervical de la preparación mediante las técnicas de doble hilo y cofia de trasferencia.. Rev. Odontol. Univ. Cid. São Paulo 2018 abr/jun 30(2) 157-168

ISSN 1983-5183

de la preparación cervical y una mayor visibilidad en el modelo de trabajo ${ }^{2,10}$, por otra parte cuando se usa inapropiadamente da como resultado una recesión gingival estéticamente inaceptable, impresiones pobres, frustración del paciente y un aumento en el costo por la repetición del trabajo protésico.

En este estudio se evaluó la nitidez de la preparación con 2 diferentes técnicas de impresión: doble hilo y cofia de transferencia con 3 materiales de impresión poliéter y 2 siliconas adición.

En un estudio realizado por Mary P. Walker y col en $2005^{19}$ mencionan que entre los materiales de impresión utilizados, el poliéter se comporta mejor tanto en condiciones de humedad como en áreas secas. Contrario al estudio de Mary P Walker nuestro estudio en encontramos mejor comportamiento de las siliconas por adición (grupos 1 y 5), que el poliéter (grupo 2).

En la escala 3 (Mal) los grupos de estudio 1,5 y 6 se comportaron mejor numéricamente que los grupos 2,3 y 4 por lo que podemos decir que las técnicas doble hilo con siliconas por adición tuvieron un mejor comportamiento, que la técnica de cofia con siliconas.

Jokstad ${ }^{16}$ en su estudio en 1999 al igual que Kumbuloglu ${ }^{20}$ en 2007 mencionan que no existe una diferencia entre la utilidad de hilos impregnados con epinefrina o cualquier otra sustancia e hilos secos sin impregnar, sin embargo se recomienda descartar a los pacientes que tengan alguna afección cardiaca ya que estos vasoconstrictores pudieran causar arritmias, mientras que Wostmann ${ }^{21}$ en 2008 afirma que la utilización de algún agente hemostático es eficaz en la reducción de fluido cervicular por lo que los primeros dos autores coinciden con nuestro estudio ya que no utilizamos ningún hemostático en el hilo.

Johnson ${ }^{22}$ en 2010 explica que no hay una gran diferencia entre la toma de impresión con cucharillas completas y con cucharillas parciales sin embargo se debe tener conocimiento sobre las indicaciones de cada una, a su vez él explica que no hay necesidad de tomar una impresión completa si únicamente se va a tomar impresión de un diente, además se ahorra material de impresión y se podría tener un mejor control de material por utilizarse en pequeñas cantidades, mientras que las cucharillas completas están indicadas para impresiones múltiples, esta será otra razón para expandir nuestra muestra y corroborar si esto es correcto ya que en nuestro estudio se utilizaron cucharillas completas.

\section{CONCLUSIÓN}

Bajo las condiciones de este estudio y los resultados obtenidos en este indican que la técnica de doble hilo obtuvo mejores resultados numéricos comparada con la técnica de cofia, aun así la diferencia no es estadísticamente significativa. En relación a los materiales usados los resultados fueron muy similares en cuanto a la reproducción del detalle y nitidez de la preparación cervical, sin embargo el clínico deberá tener un conocimiento de las ventajas y desventajas de cada uno de los materiales y técnicas así como su entrenamiento para elegir el material de impresión en cada caso en particular. Es importante dejar abierta la línea de investigación a futuros estudios. 
OJEDA GUTIÉRREZ F, MARQUEZ PRECIADO R, RAMIREZ GONZÁLEZ JH, ROJAS MUÑOZ NAM, MARTÍNEZ MARTÍNEZ RE, SÁNCHEZ ROBLES PR, GONZALEZ CORREA R. Estudio comparativo de tres materiales de impresión en su capacidad para reproducir el margen cervical de la preparación mediante las técnicas de doble hilo y cofia de trasferencia.. Rev. Odontol. Univ. Cid. São Paulo 2018 abr/jun 30(2) 157-168

ISSN 1983-5183

\section{BIBLIOGRAFÍA}

1. TANNAMALA PK, Azhagarasan NS, Shankar KC. Evaluation of marginal gap of Ni-Cr copings made with conventional and accelerated casting techniques. Indian journal of dental research : official publication of Indian Society for Dental Research 2013 Jan-Feb;24(1):147-8.

2. Reiman MB. Exposure of subgingival margins by nonsurgical gingival displacement. The Journal of Prosthetic Dentistry 1976 1976/12/01/;36(6):649-54.

3. Baharav H, Laufer BZ, Langer Y, Cardash HS. The effect of displacement time on gingival crevice width. The International journal of prosthodontics 1997 May-Jun;10(3):248-53.

4. Shillingburg H, Hobo S, Whitsett L. Fundamentos de prostodoncia fija. Barcelona: Quintessence; 2002.

5. Wostmann B, Rehmann P, Trost D, Balkenhol M. Effect of different retraction and impression techniques on the marginal fit of crowns. Journal of dentistry $2008 \mathrm{Jul} ; 36(7): 508-12$.

6. Dabas N, Vigarniya M, Phogat S, Madan R, Malhotra P, Dabas A. Comparing two cordless impression techniques for dimensional accuracy: an in vitro study. Indian J Dental Sciences 2018 10(2):61-5.

7. Gutiérrez CR, Todescan R. Prostodoncia: procedimientos de laboratorio. In: Gutiérrez, CR. Elaboración de cofias y provicionales. México: Carlos Ripol Gutiérrez; 1983. p. p. 81-878.

8. Fitzig S, Feder D, Marshak B, Orstein M. An improved copper band impression technique. Quintessence international (Berlin, Germany: 1985) 1985 Dec;16(12):851-3.

9. Mezzomo E. Rehabilitación oral para el clínico. Venezuela: Actualidades Médico Odontológicas; 2003.

10. Child Jr. PL. Is cord still king?: tissue management for fixed prosthodontics. Dental Economics Oral Hygienics 2012 102(4):72-8.

11. Francisco RLC, Roberto MM. Consideraciones ideales en la toma de impresión dental. Rev ADM 2001 LVIII(5):183-90.

12. Azzi R, Tsao TF, Carranza FA, Kenney EB. Comparative study of gingival retraction methods. J Prosthetic Dentistry 1983 50(4):561-5.

13. Ruel J, Schuessler PJ, Malament K, Mori D. Effect of retraction procedures on the periodontium in humans. J Prosthet Dent 1980 Nov;44(5):508-15.

14. Mansueto MA, Abdulkarim HA, Thabet WR, Haney SJ. The chamfer finish line: preclinical student performance using different bur designs. Journal of dental education 2010 Jun;74(6):6127.

15. Pegoraro LF. Prótesis fija. In: Pegoraro, LF. Impresiones y modelos de trabajo. Porto Alegre: Artes Médicas; 2001. p. p. 151-72. 
16. Jokstad A. Clinical trial of gingival retraction cords. J Prosthet Dent 1999 Mar;81(3):258-61.

17. Wassell RW, Barker $D$, Walls AW. Crowns and other extra-coronal restorations: impression materials and technique. British dental journal 2002 Jun 29;192(12):679-84, 87-90.

18. Anusavice KJ. Phillips ciencia de los materiales dentales. In: Anusavice, KJ. Materiales de impresión elastómeros no acuosos. Espanha: Elsevier España; 1998. p. p.143-83.

19. Walker MP, Petrie CS, Haj-Ali R, Spencer P, Dumas C, Williams K. Moisture effect on polyether and polyvinylsiloxane dimensional accuracy and detail reproduction. Journal of prosthodontics : official journal of the American College of Prosthodontists 2005 Sep;14(3):158-63.

20. Kumbuloglu O, User A, Toksavul S, Boyacioglu H. Clinical evaluation of different gingival retraction cords. Quintessence international (Berlin, Germany: 1985) 2007 Feb;38(2):e92-8.

21. Wostmann B, Rehmann P, Balkenhol M. Influence of different retraction techniques on crevicular fluid flow. The International journal of prosthodontics 2008 May-Jun;21(3):215-6.

22. Johnson GH, Mancl LA, Schwedhelm ER, Verhoef DR, Lepe X. Clinical trial investigating success rates for polyether and vinyl polysiloxane impressions made with full-arch and dualarch plastic trays. J Prosthet Dent 2010 Jan;103(1):13-22.

RECEBIDO EM 15/02/2018

ACEITO EM 04/04/2018 